\title{
ARTICLE
}

Received 25 Jun 2014 | Accepted 29 Jul 2014 | Published 11 Sep $2014 \quad$ DOl: 10.1038/ncomms5836

\section{Principle of direct van der Waals epitaxy of single-crystalline films on epitaxial graphene}

\author{
Jeehwan Kim ${ }^{1, \star}$, Can Bayram ${ }^{1, \star}$, Hongsik Park${ }^{1}$, Cheng-Wei Cheng ${ }^{1}$, Christos Dimitrakopoulos ${ }^{1, \dagger}$, \\ John A. Ott ${ }^{1}$, Kathleen B. Reuter ${ }^{1}$, Stephen W. Bedell ${ }^{1} \&$ Devendra K. Sadana ${ }^{1}$
}

There are numerous studies on the growth of planar films on $s p^{2}$-bonded two-dimensional (2D) layered materials. However, it has been challenging to grow single-crystalline films on 2D materials due to the extremely low surface energy. Recently, buffer-assisted growth of crystalline films on 2D layered materials has been introduced, but the crystalline quality is not comparable with the films grown on $s p^{3}$-bonded three-dimensional materials. Here we demonstrate direct van der Waals epitaxy of high-quality single-crystalline GaN films on epitaxial graphene with low defectivity and surface roughness comparable with that grown on conventional $\mathrm{SiC}$ or sapphire substrates. The $\mathrm{GaN}$ film is released and transferred onto arbitrary substrates. The post-released graphene/SiC substrate is reused for multiple growth and transfer cycles of GaN films. We demonstrate fully functional blue light-emitting diodes (LEDs) by growing LED stacks on reused graphene/SiC substrates followed by transfer onto plastic tapes.

\footnotetext{
${ }^{1}$ IBM T. J. Watson Research Center, 1101 Kitchawan Road, Yorktown Heights, New York 10598, USA. * These authors contributed equally to this work. $\dagger$ Present address: Department of Chemical Engineering, University of Massachusetts Amherst, 686 N. Pleasant St., Amherst, Massachusetts 01003, USA Correspondence and requests for materials should be addressed to J.K. (email: jeehwkim@us.ibm.com) or to C.B. (email: cbayram@us.ibm.com) or to H.P. (email: hpark@us.ibm.com).
} 
an der Waals epitaxy (vdWE) can allow crystalline growth on two-dimensional (2D) materials with no requirement to satisfy lattice mismatch between the growing materials and underlying 2D materials, resulting in reduced defect density $^{1,2}$. Recently, this concept has drawn renewed attention with current substantial advancement of research on 2D layered materials $^{3,4}$. If one can grow $2 \mathrm{D}$ blanket epitaxial films on $2 \mathrm{D}$ materials, it will open up a new opportunity to beyond current extensive research on large-scale fabrication of crystallographically aligned $2 \mathrm{D}$ heterostructures ${ }^{3,4}$. If one can grow $s p^{3}$-bonded threedimensional (3D) epitaxial films on 2D materials, it will allow transfer of single-crystalline semiconductor films due to weak van der Waals interaction between $2 \mathrm{D}$ materials and overlaying layers ${ }^{5}$.

However, suppressed nucleation of adatoms on the surface of $s p^{2}$-bonded $2 \mathrm{D}$ materials limits the large-area single-crystalline growth $^{2,6-10}$. Therefore, most of the attempts to grow blanket films on $2 \mathrm{D}$ materials resulted in nonplanar film morphologies. For example, direct growth of $2 \mathrm{D}$ on $2 \mathrm{D}$ materials forms flakes such as $\mathrm{MoS}_{2}$ flakes on graphene ${ }^{2,11}$, whereas direct epitaxy of $s p^{3}$-bonded $3 \mathrm{D}$ materials on $2 \mathrm{D}$ materials causes the formation of $3 \mathrm{D}$ clusters such as GaN clusters on h-BN or graphene ${ }^{2,6-10}$. Recently, growth of planar 3D material films on 2D materials was demonstrated by employing interfacial buffers between $3 \mathrm{D}$ and $2 \mathrm{D}$ materials to promote nucleation of $3 \mathrm{D}$ materials on $2 \mathrm{D}$ materials such as $\mathrm{GaN}$ film growth on $\mathrm{ZnO}$ nanowalls/ graphene ${ }^{9,12,13}$ and GaN film growth on AlN/h-BN (or graphene $)^{10,14}$. Even with the interfacial buffer, however, the quality of $\mathrm{GaN}$ films grown on $2 \mathrm{D}$ materials is significantly worse than that on a conventional $\mathrm{SiC}$ or sapphire substrate. In the best case, $\mathrm{GaN}$ on AlN/h-BN, the measured defect density $\left(1.6 \times 10^{10} \mathrm{~cm}^{-2}\right)$ is greater than that from conventional GaN epitaxial films grown on sapphire by more than one order of magnitude and surface roughness was not as low as that of conventional GaN epitaxial films on sapphire ${ }^{10,15}$. Having considering the fact that an AlN buffer has also been an essential component for conventional growth of high-quality GaN films on sapphire substrates ${ }^{15}$, different growth strategies are required for the epitaxy on 2D materials. Moreover, buffered growth is not applicable for the growth of $2 \mathrm{D}$ on $2 \mathrm{D}$ materials where direct contact of those materials makes device functions ${ }^{3,4}$ and also for the growth of vertical electronic devices that require formation of initial films with high electrical conductivity ${ }^{15}$.
Here we demonstrate direct growth of high-quality singlecrystalline films on 2D materials. We choose epitaxial graphene grown on a $\mathrm{SiC}$ substrate as a template for growing singlecrystalline films because it retains a unique orientation over an entire substrate and it contains the periodic nucleation sites at the step edges ${ }^{16,17}$. We perform direct vdWE of single-crystalline $\mathrm{GaN}$ films on epitaxial graphene on $\mathrm{SiC}$ substrates by careful control of growth kinetics. Even without using any buffer layers, GaN crystalline quality comparable with that typically obtained via conventional AlN-buffer-assisted GaN epitaxy on $\mathrm{SiC}$ or sapphire substrates is obtained by overcoming substantial lattice mismatch of $\sim 23 \%$ between graphene and GaN. Measured root mean square (RMS) roughness is $3 \AA$ and defect density is as low as $4 \times 10^{8} \mathrm{~cm}^{-2}$. The entire high-quality single-crystalline $\mathrm{GaN}$ films are then released from the graphene and transferred on arbitrary substrates. Owing to precise release of GaN from the surface of graphene with no remaining $\mathrm{GaN}$ on the graphene, demonstration of multiple cycles of growth and transfer of $\mathrm{GaN}$ films is enabled by reusing the single graphene/SiC substrate with no requirement of post-release surface treatment. Additionally, thanks to the atomistic smoothness of released GaN surface, direct bonding of released $\mathrm{GaN}$ onto Si substrate is enabled. In this study, we introduce the epitaxial graphene as a reusable platform for multiple transfers of high-quality single-crystalline layers and offer the general principle of direct vdWE on 2D materials which may also be applicable to other materials systems such as $2 \mathrm{D}$ heterostructures.

\section{Results}

Graphene-based layer transfer graphene. A schematic of our graphene-based layer transfer process is shown in Fig. 1 and the process consists of the following steps: formation of epitaxial graphene by self-limiting graphitization of a vicinal (0001) $\mathrm{SiC}$ substrate $^{16,17}$ (Fig. 1a), epitaxy of single-crystalline GaN films on graphene/SiC substrates by taking advantage of facilitated nucleation at vicinal steps (Fig. 1b), release of entire $\mathrm{GaN}$ films from the graphene surface by using a stressor metal $(\mathrm{Ni})$ and the flexible handling layer (thermal release tape) ${ }^{18,19}$ (Fig. 1c,d), transfer of the released $\mathrm{GaN}$ to host substrates (Fig. 1e,f) and multiple growths and transfers of the GaN films on/from the original graphene/SiC substrate.

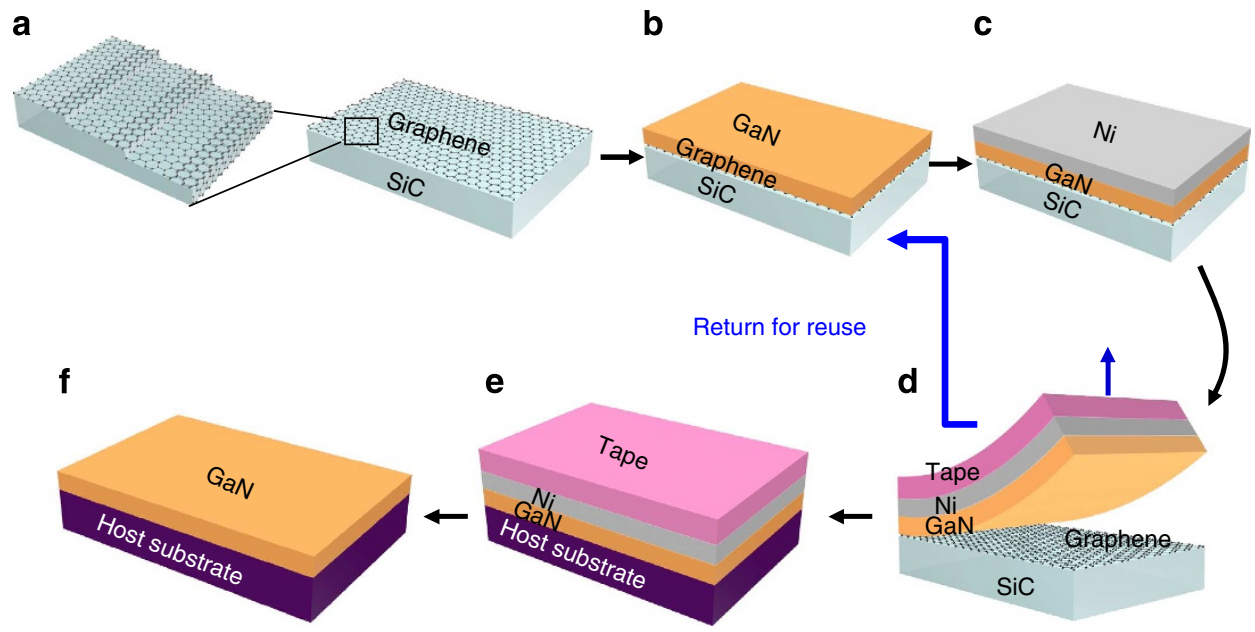

Figure 1 | Schematic of a method for growing/transferring single-crystalline thin films on/from epitaxial graphene. (a) Graphitization of a SiC substrate to form epitaxial graphene. (b) Epitaxial growth of $\mathrm{GaN}$ on graphene. (c) Deposition of a stressor layer (Ni). (d) Release of GaN from the substrate with a handling tape. (e) Transfer of the released GaN/Ni/tape stack on a host substrate. (f) Removal of the tape and Ni by thermal release and wet etching, leaving a GaN film on the host substrate. 
a

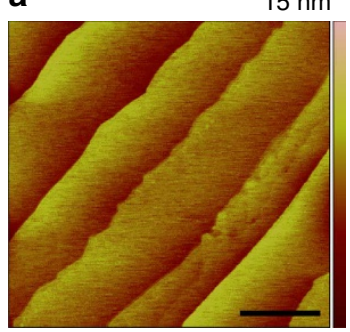

b

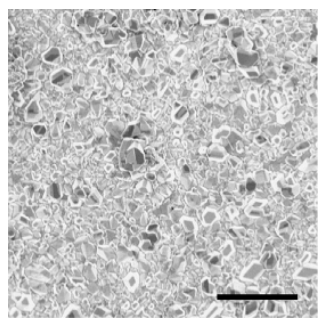

c

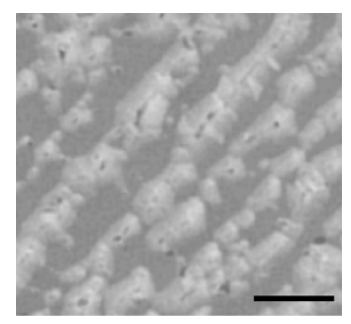

d

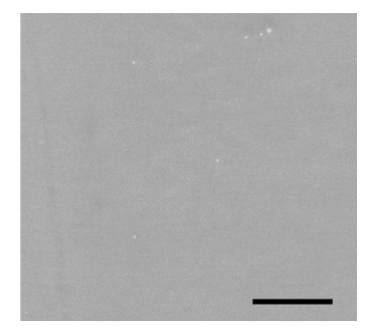

e

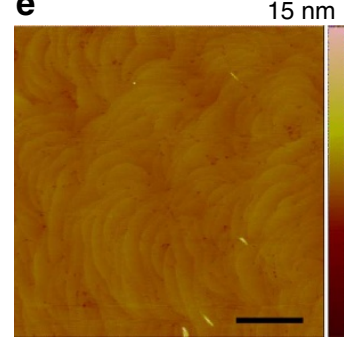

Figure 2 I Surface morphology of a graphene/SiC substrate and GaN films grown on graphene. (a) AFM image of the surface of a graphitized SiC substrate (scale bar, $10 \mu \mathrm{m}$ ). (b-d) Plan-view SEM images of GaN films grown on graphene (scale bars, $10 \mu \mathrm{m}$ ) by (b) the conventional two-step growth (nucleation at $580^{\circ} \mathrm{C}$ and growth at $1,150{ }^{\circ} \mathrm{C}$ ), (c) one-step growth at $1,100^{\circ} \mathrm{C}$, and (d) modified two-step growth (nucleation at $1,100{ }^{\circ} \mathrm{C}$ and growth at $1,250^{\circ} \mathrm{C}$ ). (e) AFM image of GaN films grown by modified two-step growth showing atomistically smooth surface with RMS roughness of $3 \AA$ (scale bar, $1 \mu \mathrm{m}$ ).

vdWE of planar single crystals on graphene. Figure 2a shows the atomic force microscopy (AFM) surface topology of the epitaxial graphene on a SiC substrate after the graphitization. The size of the vicinal steps of the $\mathrm{SiC}$ surface was increased by the step bunching during the graphitization ${ }^{17}$. The measured step height and terrace width were $5-10 \mathrm{~nm}$ and $5-10 \mu \mathrm{m}$, respectively. Continuous films are achievable if $\mathrm{GaN}$ nuclei can be preferentially formed first along the periodic step edges and GaN nuclei then advance laterally to coalesce. We performed the growth of $\mathrm{GaN}$ on epitaxial graphene by using metalorganic chemical vapour deposition (MOCVD) with following conditions: the conventional two-step growth used for GaN epitaxy on sapphire or $\mathrm{SiC}$ (nucleation at $580^{\circ} \mathrm{C} /$ growth at $1,150{ }^{\circ} \mathrm{C}$ ) ${ }^{15,20,21}$, one-step growth at $1,100^{\circ} \mathrm{C}$ and modified two-step growth (nucleation at $1,100^{\circ} \mathrm{C} /$ growth at $1,250^{\circ} \mathrm{C}$ ). Figures $2 \mathrm{~b}-\mathrm{d}$ show plan-view scanning electron microscopy (SEM) images of $\mathrm{GaN}$ films grown on graphene by the above three conditions, respectively. The conventional two-step growth (nucleation at $580^{\circ} \mathrm{C} /$ growth at $1,150^{\circ} \mathrm{C}$ ) formed $3 \mathrm{D}$ faceted $\mathrm{GaN}$ clusters (Fig. 2b), whereas one-step growth at $1,100^{\circ} \mathrm{C}$ resulted in the formation of continuous $\mathrm{GaN}$ stripes ( 5-10 $\mu \mathrm{m}$ apart) aligned along the $\mathrm{SiC}$ vicinal steps (Fig. 2c). We speculated that the formation of such faceted clusters from the conventional two-step growth was attributed to the limited atomic mobility at $580^{\circ} \mathrm{C}$ together with suppressed nucleation on the graphene surface resulting in the low density of nuclei randomly formed on the terrace $^{22,23}$. The formation of stripe patterns from one-step growth at $1,100{ }^{\circ} \mathrm{C}$ implies that the increased atomic mobility allows adatoms to nucleate at the energetically favourable step edges $^{22,23}$. On the other hand, the modified two-step growth (nucleation at $1,100^{\circ} \mathrm{C}$, growth at $1,250^{\circ} \mathrm{C}$ ) resulted in the formation of continuous and smooth GaN films (Fig. 2d). This could be attributed to the faster lateral growth at an increased growth temperature of $1,250^{\circ} \mathrm{C}$ from the $\mathrm{GaN}$ nuclei along the periodic terrace edges formed at $1,100^{\circ} \mathrm{C}$ (ref. 24). A root mean square (RMS) roughness of $3 \AA$ was measured from the surface of GaN formed by the modified two-step growth by using AFM (Fig. 2e). Note that no growth occurs when the one-step growth was performed at $1,250^{\circ} \mathrm{C}$ due to reduced sticking coefficient (see Supplementary Fig. 1).

Detailed structural analysis of the GaN grown on epitaxial graphene was performed using transmission electron microscopy (TEM). As it is known that graphene formed on the SiC surface via $\mathrm{Si}$ sublimation retains its unique orientation throughout an entire SiC substrate ${ }^{16,17,19}$, formation of single-crystalline GaN films was expected if the GaN films were epitaxially grown. The low-magnification TEM image (Fig. 3a) shows that a uniform $2.5-\mu \mathrm{m}$-thick $\mathrm{GaN}$ film was grown on graphene/SiC. The highresolution TEM (HRTEM) image shows well aligned, ordered crystal lattices of GaN, graphene and $4 \mathrm{H}-\mathrm{SiC}$ (Fig. 3b). Figure 3c shows selected area electron diffraction patterns taken from GaN and $\mathrm{SiC}$, and across the stack of $\mathrm{GaN} /$ graphene/SiC. The diffraction patterns from $\mathrm{GaN}$ and $\mathrm{SiC}$ indicate planes of a single-crystalline wurtzite structure and they are well aligned (see magnified view in the last image in Fig. $3 c$ to see the alignment). This average proves successful epitaxial growth of $\mathrm{GaN}$ on a graphene/SiC substrate. The diffraction from graphene was also observed with a reduced aperture size to $300 \mathrm{~nm}$ to focus on the interface, where the graphene patterns appear as streaks due to the lack of plane (see Supplementary Fig. 2). To evaluate the crystal quality of the epitaxially grown $\mathrm{GaN}$ on graphene, we analysed the defectivity of the GaN film by using plan-view TEM. The average density of threading dislocations over a total inspected area of $\sim 30 \mu \mathrm{m}^{2}$ was approximately $1 \times 10^{9} \mathrm{~cm}^{-2}$. Figure $3 \mathrm{~d}$ shows representative plan-view TEM images. This value is in a good agreement with the density of surface pits in the AFM image (Fig. 2e). Notably, this density is in the comparable range with that of AlN-buffer-assisted GaN films grown on the conventional substrates, sapphire or $\mathrm{SiC}$ by using MOCVD $\left(5 \times 10^{8} \mathrm{~cm}^{-2}-8 \times 10^{9} \mathrm{~cm}^{-2}\right)$ (refs 25-27). Such low dislocation density could be obtained even with $\sim 23 \%$ mismatch between $\mathrm{GaN}$ and graphene and even without employing AlN buffers, thanks to the successful vdWE of GaN on graphene.

To determine the macroscopic orientation and the crystal structure, we also performed high-resolution X-ray diffraction (HRXRD) with 2theta/omega scan and azimuthal off-axis phi scans (Fig. 3e,f, respectively). The XRD spectrum with the 2theta/omega scan represents only two peaks corresponding to single-crystalline wurtzite (0002) $\mathrm{GaN}$ and (0004) 4H-SiC (Fig. 3e). We have performed multiple scans across the sample and any peaks other than the (0002) GaN peak were not identified. The wider XRD spectrum is shown in Supplementary Fig. 3. The full-width half maximum of the GaN (0002) peak $\left(=0.06^{\circ}\right)$ is in the similar range to that measured from GaN grown on sapphire or $\mathrm{SiC}^{25-27}$ (see Supplementary Table 1). The phi scan was performed by rotating the sample around its surface normal (Fig. 3f). The sixfold symmetry of the diffraction peaks of $\mathrm{GaN}$ (105) with $60^{\circ}$ intervals indicates that the $\mathrm{GaN}$ is a single-crystalline wurtzite phase. Large-area electron backscatter diffraction maps also confirmed the single crystallinity of GaN grown on epitaxial graphene (see Supplementary Fig. 3b). It should be noted that CVD-grown graphene on $\mathrm{Cu}$ foils cannot be employed for obtaining single-crystalline films as the graphene itself is polycrystalline $\mathrm{e}^{12,13}$.

Release/transfer of single-crystalline GaN on graphene. The TEM and HRXRD analysis conclusively shows that a high-quality 
a

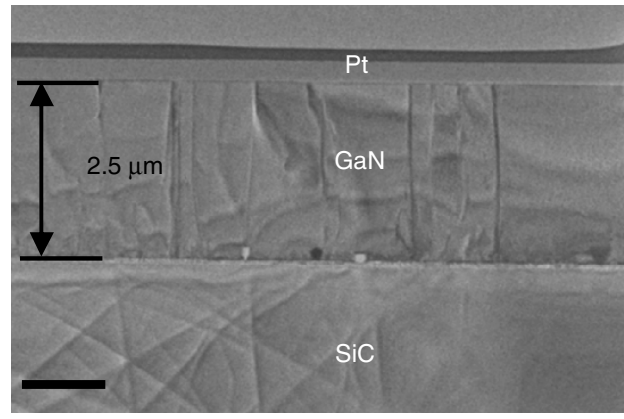

b

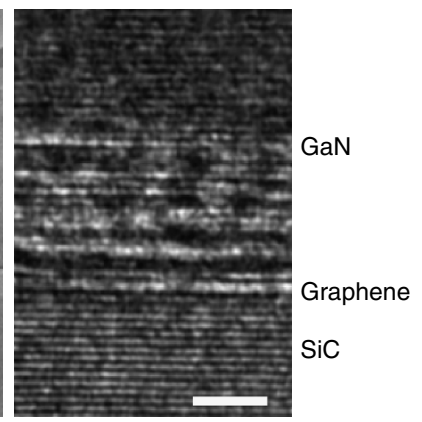

C
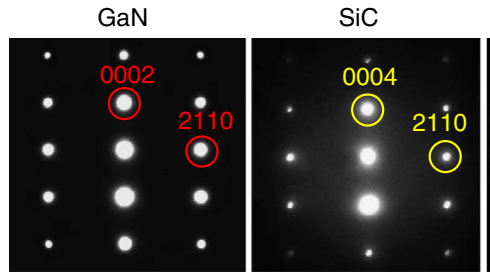

GaN/graphene/SiC
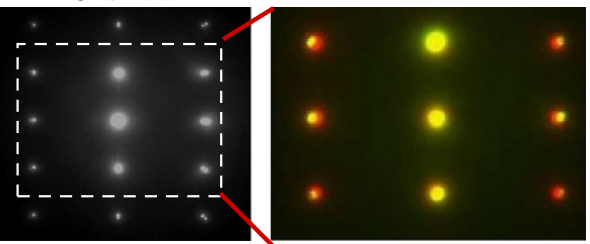

d
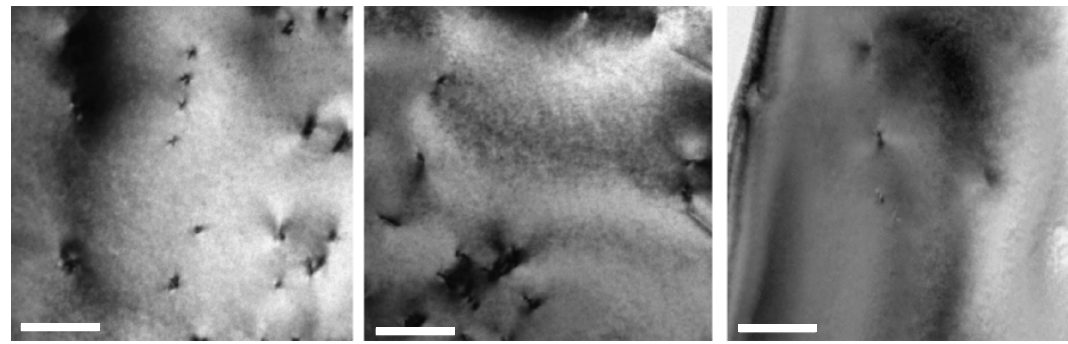

e
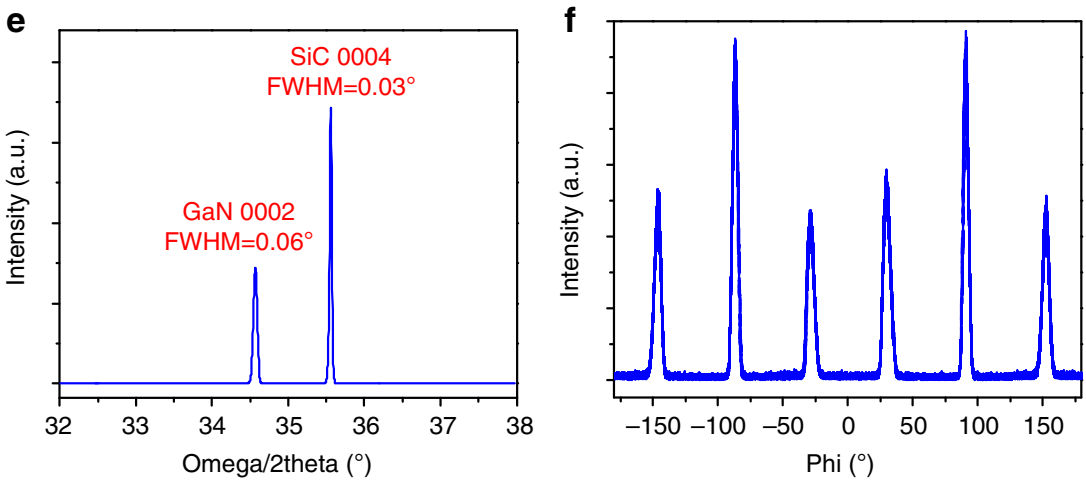

Figure 3 | TEM and XRD analyses on single crystallinity of GaN films grown on epitaxial graphene. (a) Low-magnification cross-sectional TEM image of $2.5-\mu \mathrm{m}$-thick $\mathrm{GaN}$ grown on a graphene/SiC substrate (scale bar, $1 \mu \mathrm{m}$ ). (b) HRTEM taken at the interface of GaN/graphene/SiC showing well ordered and aligned crystal lattices of $\mathrm{GaN} /$ graphene/SiC (scale bar, $2 \mathrm{~nm}$ ). (c) Selected area electron diffraction patterns from GaN, SiC, and the interface of $\mathrm{GaN} / g r a p h e n e / \mathrm{SiC}$. All diffraction patterns show the planes of a single-crystalline wurtzite structure. The foil plane vertically aligned with the beam is (0110). The last diffraction pattern is the sum of the $\mathrm{GaN}$ and $\mathrm{SiC}$ patterns shown. The yellow dots and red dots that appear in a magnified view correspond to $\mathrm{SiC}$ and $\mathrm{GaN}$, respectively, indicating excellent alignment of $\mathrm{GaN}$ and $\mathrm{SiC}$. (d) Plan-view TEM images of released $\mathrm{GaN}$ from the graphene/SiC substrate (scale bar, $0.2 \mu \mathrm{m}$ ). The density of threading dislocations (from left image to right one) is $2.3 \times 10^{9} \mathrm{~cm}^{-2}, 1.5 \times 10^{9} \mathrm{~cm}^{-2}$ and $0.4 \times 10^{9} \mathrm{~cm}^{-2}$. (e) HRXRD omega/2theta scan. Peaks correspond to wurtzite (0002) GaN and (0004) $4 \mathrm{H}-\mathrm{SiC}$. (f) HRXRD azimuthal off-axis phi scan. Peaks of $\mathrm{GaN}(105)$ at $60^{\circ}$ intervals represent the sixfold symmetry of a single-oriented wurtzite crystal.

single-crystalline GaN film can be grown on an epitaxial graphene surface. Therefore, high technical impact is expected if these highquality GaN films can be transferred onto the foreign substrates of interest. Release of an overlaying $\mathrm{GaN}$ epitaxial film can be initiated either at the GaN/graphene or graphene/SiC interface if the strain energy of the GaN film induced by external stress reaches the van der Waals-bonding energy of the GaN/graphene or graphene/SiC interface. We applied $\mathrm{Ni}$ as a stressor layer on $\mathrm{GaN}$ because strain energy in GaN can be effectively induced by high-internal stress of $\mathrm{Ni}$ (that is, $500 \mathrm{MPa}-1 \mathrm{GPa})^{18,19}$. The $2.5-\mu \mathrm{m}$-thick GaN film was released from the substrate by a $2-\mu \mathrm{m}$-thick $\mathrm{Ni}$ stressor as shown in the cross-sectional SEM (XSEM) image (Fig. 4a). Thermally released tape was applied on $\mathrm{Ni}$ as a handling layer. The interface separation shown in the figure occurred by sample cleaving for the XSEM analysis. Entire GaN epilayer was released from the graphitized substrate with the area of $\sim 1 \mathrm{~cm}^{2}$ (see Supplementary Fig. 4)-limited by the size of our MOCVD reactor. In principle, a wafer-scale GaN release is 
a

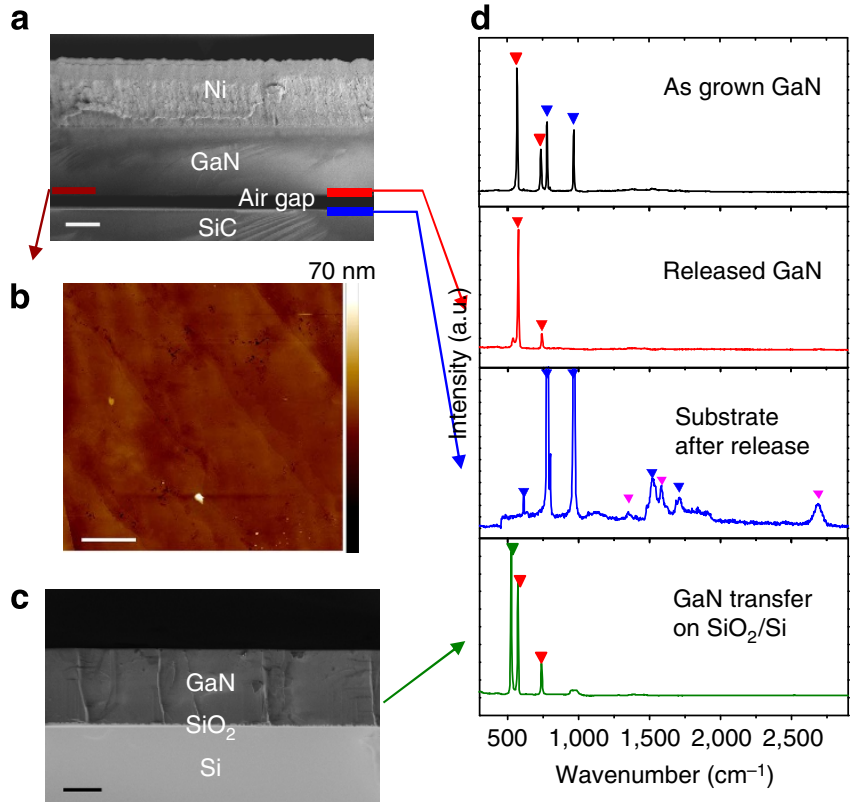

e
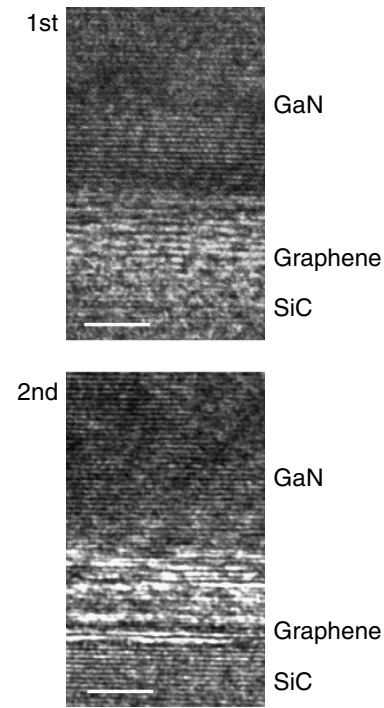

Figure 4 | Analysis on transferability of GaN from epitaxial graphene and reusability of graphene/SiC substrates. (a) XSEM image of GaN on a graphene/SiC substrate after Ni stressor deposition (scale bar, $1 \mu \mathrm{m}$ ). Separation of the interface between a GaN film and a graphene/SiC substrate is displayed. (b) AFM images taken at the released surface of GaN films (scale bar, $3 \mu \mathrm{m}$ ). The terrace patterns appear as those observed from the graphitized SiC substrate. (c) XSEM image of $\mathrm{GaN}$ films directly transferred on a $\mathrm{SiO}_{2} / \mathrm{Si}$ substrate (scale bar, $1 \mu \mathrm{m}$ ). (d) Raman spectra taken from an as-grown GaN film on a graphene/SiC substrate, released GaN films on the tape, remaining substrate after GaN release and GaN film transferred on $\mathrm{SiO}_{2} / \mathrm{Si}$ substrate. Red, blue, purple and green inverted triangles correspond to $\mathrm{GaN}, \mathrm{SiC}$, graphene and $\mathrm{Si}$, respectively. (e) HRTEM taken at the interface of GaN/graphene/SiC (scale bar, $2 \mathrm{~nm}$ ). GaN grown on a fresh graphene/SiC substrate is shown on the top, and GaN grown on a reused graphene/ $\mathrm{SiC}$ substrate after the 1st GaN release is shown on the bottom. They both show comparable crystal order/alignment and graphene still exists on the $\mathrm{SiC}$ substrate after $\mathrm{GaN}$ release.

possible. The topology of the released surface of $\mathrm{GaN}$ films imaged by AFM (Fig. 4b) shows similar terraces to those observed on the surface of an original graphitized $\mathrm{SiC}$ substrate (Fig. 2a), implying the removal of entire GaN films precisely from the surface of the graphitized $\mathrm{SiC}$ substrate. The flat surface of the released GaN layer (RMS roughness of $\sim 5 \AA$ measured from AFM image in Supplementary Fig. 5) allowed us to bond the released GaN film directly onto (100) Si substrates with a 90-nm $\mathrm{SiO}_{2}$ layer $^{28}$. As shown in the XSEM image in Fig. 4c, we could achieve the unique structure of $\mathrm{GaN}$ on insulator which can be applied for a hybrid GaN and (001) Si co-integration. The formation of excellent bonding of the transferred $\mathrm{GaN}$ on $\mathrm{SiO}_{2}$ was also verified by TEM investigation on the interface (see Supplementary Fig. 6). No air gap was detected throughout low magnification and high-resolution TEM analyses. The transferred $\mathrm{GaN}$ on insulator shows perfect single-crystalline diffraction patterns indicating no degradation of crystalline quality during the transfer process (see Supplementary Fig. 6).

To accurately determine the released interface, we analysed Raman spectra measured from the released surface of $\mathrm{GaN}$ films and the surface of the remaining substrate (Fig. 4d). The Raman peaks corresponding to graphene was clearly observed from the surface of the post-released $\mathrm{SiC}$ substrate, but only GaN peaks were observed from the released film without any peaks corresponding to graphene (Fig. $4 \mathrm{~d}$ ). The uniform coverage of graphene on the surface of the post-released $\mathrm{SiC}$ substrate was observed by mapping the Raman intensity of graphene $2 \mathrm{D}$ peak (see Supplementary Fig. 7). The Raman analysis clearly indicates that $\mathrm{GaN}$ is released from the surface of graphene instead of being released together with graphene. This result also implies that the bonding energy of GaN/graphene is lower than that of graphene/ $\mathrm{SiC}(\sim 106 \mathrm{meV} \text { per atom })^{19}$. We repeated the sequence of $\mathrm{GaN}$ epitaxial growth on the post-released graphene/SiC substrate and the transfer of the GaN layer. Well ordered, aligned GaN crystal lattices on graphene were observed for the first growth and multiple growth of GaN by HRTEM. As an example, we have shown HRTEM images of the GaN/graphene/SiC interface after the first GaN growth and that after the second GaN growth on the graphene/ $\mathrm{SiC}$ substrate used for the first growth/transfer (see Fig. 4e).

Growth/release of thin-film blue LED on graphene. To demonstrate further functionality of our graphene-based single-crystalline multiple-growth/transfer technique, we have performed epitaxial growth of a visible LED stack on a recycled graphene/SiC substrate (previously used 'three times'). The visible LED stack is composed of three periods of III-nitride multi-quantum wells (InGaN well and $\mathrm{GaN}$ barrier) sandwiched between $\mathrm{p}-\mathrm{GaN}$ and $\mathrm{n}-\mathrm{GaN}$ layers (see cross-sectional TEM images of a LED stack on graphene/SiC in Fig. 5a,b). The HRTEM image taken at the GaN/graphene/SiC interface shows well aligned, ordered $\mathrm{GaN}$ crystal lattice on a recycled graphene/ SiC substrate (see Fig. 5c). The measured RMS surface roughness was $4 \AA$. The result of HRXRD also verified the single crystallinity of a InGaN/GaN LED heterostructure on a recycled graphene/SiC substrate as the spectra reveal only two major peaks corresponding to single-crystalline wurtzite (0002) GaN and (0004) $4 \mathrm{H}-\mathrm{SiC}$, and satellite peaks corresponding to the multi-quantum wells (see Supplementary Fig. 8). The full-width half maximum of the GaN (0002) peak is $\sim 0.06^{\circ}$, which is same as that of GaN grown on a fresh graphene/ $\mathrm{SiC}$ substrate. XRD simulation shown in Supplementary Fig. 8 suggests an average indium content of $30 \%$ in the $3.5 \mathrm{~nm}$-thick InGaN quantum wells neighbouring the $\mathrm{GaN}$ barriers which is required for emission in the blue spectrum.

To form an electrical contact on the $\mathrm{p}-\mathrm{GaN}$, we have deposited thin $\mathrm{Ni} / \mathrm{Au}(5 \mathrm{~nm} / 5 \mathrm{~nm})$ and annealed at $500{ }^{\circ} \mathrm{C}$ for $10 \mathrm{~min}$. 
a

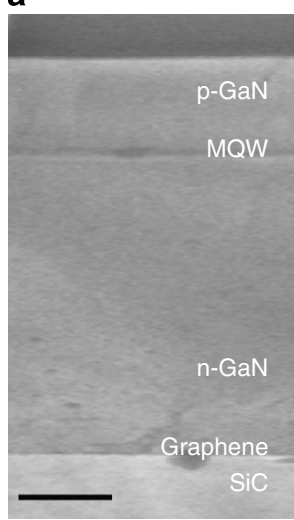

$\mathbf{e}$

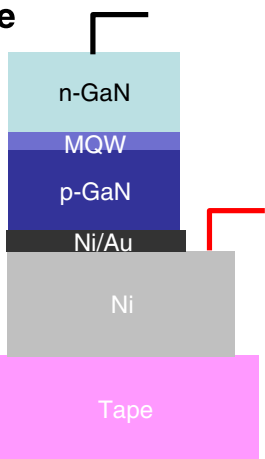

b

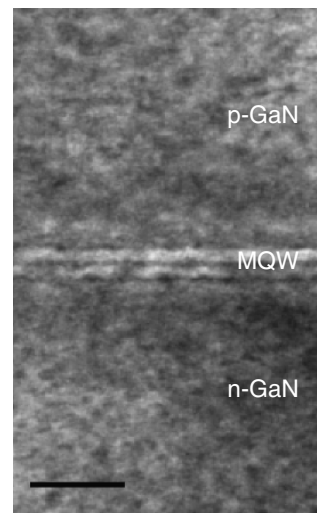

f

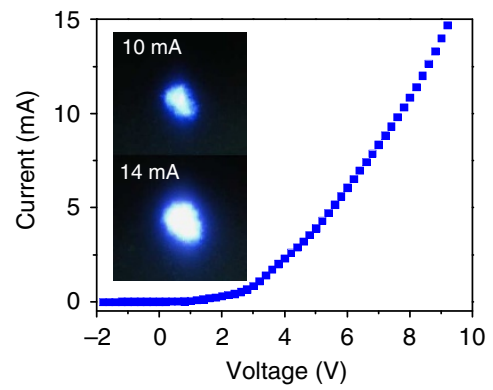

c

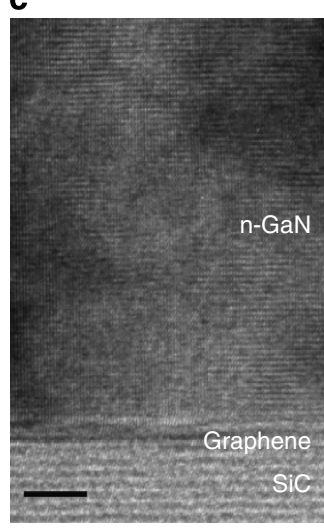

d

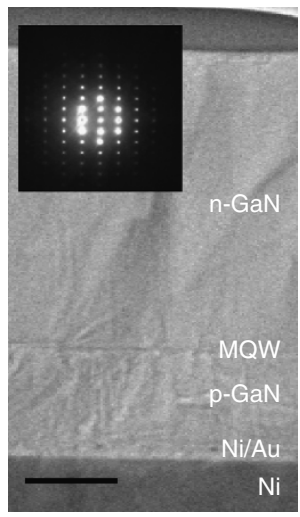

g

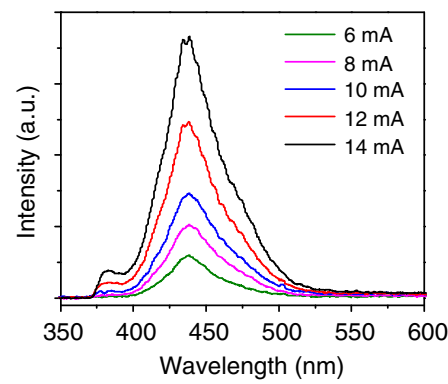

Figure 5 | Fabrication of GaN blue LED transferred on the tape. (a) Cross-sectional TEM image of LED stacks (p-GaN/MQW/n-GaN) on a graphene/SiC substrate (scale bar, $1 \mu \mathrm{m}$ ). (b) HRTEM image magnified at MQW (scale bar, $100 \mathrm{~nm}$ ). (c) HRTEM image magnified at a GaN/graphene/SiC interface (scale bar, $5 \mathrm{~nm}$ ). (d) Cross-sectional TEM image of a released LED stack from a graphene/SiC substrate: $\mathrm{n}-\mathrm{GaN} / \mathrm{MQW} / \mathrm{p}-\mathrm{GaN} / \mathrm{Ni}$ (scale bar, $1 \mu \mathrm{m}$ ). A selected area electron diffraction pattern from $\mathrm{p}-\mathrm{GaN} / \mathrm{MQW} / \mathrm{n}-\mathrm{GaN}$ is displayed in an inset. (e) Schematic of a transferred visible LED device on the tape. (f) I-V characteristic of a transferred LED stack measured by applying positive bias on Ni and negative bias on n-GaN. The pictures of the LED-emitting blue light are displayed in an inset. (g) Electroluminescence (EL) spectra of a transferred LED stack taken as a function of injection current.

The entire LED device stack was released by a Ni stressor/ handling tape as described earlier (see cross-sectional TEM images of a released LED stack on Ni in Fig. 5d). A selected area electron diffraction pattern taken from the released LED stack indicates a well-aligned single crystal (see inset of Fig. 5d). We have directly probed as-released LED stack on Ni/tape as shown in the schematic of the device structure (see Fig. 5e). A diodic $I-V$ curve was obtained (Fig. 5f). The blue light emission from the released LED stack was visually observed (inset of Fig. 5f) and electroluminescence was recorded (Fig. $5 \mathrm{~g}$ ). Electroluminescence spectra confirmed the light emission peaking at $\lambda \approx 440 \mathrm{~nm}$ which is typical for III-nitride blue LEDs.

\section{Discussion}

Our accomplishments, reusing a single graphene/SiC substrate for multiple transfers and direct bonding of $\mathrm{GaN}$ on $\mathrm{Si}$ substrate enabled by atomistically smooth released interface, are clear contrast to the conventional laser lift-off process, where postrelease surface treatment is essential to further repeat the growth/ lift-off cycles, and the high roughness of released surface did not allow direct bonding of released layer to the other substrate, it rather requires to apply adhesive to bond the released layers ${ }^{29,30}$.

In summary, we demonstrated direct vdWE of high-quality single-crystalline $\mathrm{GaN}$ on epitaxial graphene/SiC substrates resulting in the average dislocation density of $\sim 1 \times 10^{9} \mathrm{~cm}^{-2}$ and RMS surface roughness of $3 \AA$ which are within the range to those of AlN-buffer-assisted GaN films grown on the conventional substrates by using MOCVD $\left(5 \times 10^{8} \mathrm{~cm}^{-2}-8 \times\right.$ $10^{9} \mathrm{~cm}^{-2}$ ). Entire GaN films on graphene were released and transferred onto arbitrary substrates. The post-released graphene/ $\mathrm{SiC}$ substrate was reused for multiple growth and transfer cycles of GaN films. Fully functional blue LED was fabricated by performing epitaxial growth of an InGaN/GaN heterostructure on a recycled graphene/SiC substrate followed by release of the heterostructure.

\section{Methods}

Formation of epitaxial graphene. An epitaxial graphene with a single-crystalline orientation is grown on the $\mathrm{Si}$ face of a (0001) $4 \mathrm{H}-\mathrm{SiC}$ wafer via multistep annealing, first in $\mathrm{H}_{2}$ gas for surface etching and vicinalization, and then in $\mathrm{Ar}$ for graphitization at $1,575^{\circ} \mathrm{C}$.

Epitaxial growth of GaN on graphene/SiC. Epitaxial growth of GaN films on the graphene/SiC substrate was performed in a horizontal flow, low-pressure MOCVD reactor. A graphene/SiC substrate was baked under $\mathrm{H}_{2}$ for $>15 \mathrm{~min}$ at $>1,100^{\circ} \mathrm{C}$ for cleaning the surface. The deposition of a $2.5-\mu \mathrm{m}$-thick $\mathrm{GaN}$ layer was immediately followed at 200 mbar. Trimethylgallium, ammonia and hydrogen were used as a Ga source, nitrogen source and carrier gas, respectively. A modified two-step growth was employed to obtain flat GaN epitaxial films on the epitaxial graphene. The first step was carried out at a growth temperature of $1,100^{\circ} \mathrm{C}$ for few minutes where guided nucleation at terrace edges was promoted. The second growth step was carried out at an elevated temperature of $1,250{ }^{\circ} \mathrm{C}$ to promote the lateral growth. Vertical GaN growth rate was around $20 \mathrm{~nm} \mathrm{~min}^{-1}$.

Release/transfer of epitaxial GaN on graphene. The Ni stressor was deposited in the evaporator at a vacuum level of $1 \times 10^{-5}$ Torr. GaN was then completely released using the thermally released tape-handling layer. The remaining graphene/ $\mathrm{SiC}$ substrate is dipped into $\mathrm{FeCl}_{3}$-based solution to completely remove Ni residue for the next $\mathrm{GaN}$ regrowth. The released $\mathrm{GaN}$ film is transferred onto the Si wafer coated with $90-\mathrm{nm} \mathrm{SiO}_{2}$ by pressing down the stack of layers, followed by the removal of the thermal tape by annealing above the release temperature of $90^{\circ} \mathrm{C}$, 
and then etching the $\mathrm{Ni}$ film in a $\mathrm{FeCl}_{3}$-based solution. The above steps were repeated for multiple transfers.

TEM sample preparation. For the preparation of a plan-view TEM sample, a released stack (thermal release tape/Ni/GaN) was fixed to a copper grid. Then, the tape was thermally released followed by removal of $\mathrm{Ni}$ via Argon ion milling. The area imaged for the defectivity is the top $200 \mathrm{~nm}$ of GaN material.

Growth of LED on graphene. The material growth for the LED was carried out in a MOCVD. The first $\mathrm{n}$-GaN was grown by using modified two-step growth at $1,100-1,250^{\circ} \mathrm{C}$ followed by growing three periods of InGaN/GaN active layer at $\sim 700^{\circ} \mathrm{C}$. The active layer was then capped with a p-GaN layer deposited at $1,050^{\circ} \mathrm{C}$. The LED structure was later annealed at $1,000^{\circ} \mathrm{C}$ for $30 \mathrm{~s}$ under $\mathrm{N}_{2}$ ambient for carriers in the p-type material.

\section{References}

1. Koma, A., Sunouchi, K. \& Miyajima, T. Fabrication and characterization of heterostructures with subnanometer thickness. Microelectron. Eng. 2, 129-136 (1984).

2. Utama, M. I. B. et al. Recent developments and future directions in the growth of nanostructures by van der Waals epitaxy. Nanoscale 5, 3570-3588 (2013).

3. Geim, A. K. \& Grigorieva, I. V. Van der Waals heterostructures. Nature 499, 419-425 (2013).

4. Butler, S. Z. et al. Progress, challenges, and opportunities in two-dimensional materials beyond graphene. ACS Nano 7, 2898-2926 (2013).

5. Huang, R. Graphene: show of adhesive strength. Nat. Nanotechnol. 6, 537-538 (2011).

6. Loher, T., Tomm, Y., Pettenkofer, C. \& Jaegermann, W. Van der Waals epitaxy of three-dimensional CdS on the two-dimensional layered substrate $\mathrm{MoTe}_{2}$ (0001). Appl. Phys. Lett. 65, 555-557 (1994).

7. Loher, T. et al. Highly oriented layers of the three-dimensional semiconductor $\mathrm{CdTe}$ on the two-dimensional layered semiconductors $\mathrm{MoTe}_{2}$ and $\mathrm{WSe}_{2}$ J. Appl. Phys. 80, 5718-5722 (1996).

8. Gupta, P. et al. MOVPE growth of semipolar III-nitride semiconductors on CVD graphene. J. Cryst. Growth 372, 105-108 (2013).

9. Chung, K., Lee, C.-H. \& Yi, G.-C. Transferable GaN layers grown on ZnOcoated graphene layers for optoelectronic devices. Science 330, 655-657 (2010).

10. Kobayashi, Y., Kumakura, K., Akasaka, T. \& Makimoto, T. Layered boron nitride as a release layer for mechanical transfer of GaN-based devices. Nature 484, 223-227 (2012).

11. Shi, Y. et al. Van der Waals epitaxy of $\mathrm{MoS}_{2}$ layers using graphene as growth templates. Nano Lett. 12, 2784-2791 (2012).

12. Chung, K., Park, S. I., Baek, H., Chung, J.-S. \& Yi, G.-C. High-quality GaN films grown on chemical vapor-deposited graphene films. NPG Asia Mater. 4, e24 (2012).

13. Yoo, H., Chung, K., Park, S. I., Kim, M. \& Yi, G.-C. Microstructural defects in $\mathrm{GaN}$ thin films grown on chemically vapor deposited graphene layers. Appl. Phys. Lett. 102, 051908 (2013).

14. Nepal, N. et al. Epitaxial growth of III-nitride/graphene heterostructures for electronic devices. App. Phys. Exp. 6, 061003 (2013).

15. Liu, L. \& Edgar, J. H. Substrates for gallium nitride epitaxy. Mater. Sci. Eng. $R$ 37, 61-127 (2002)

16. Emtsev, K. V. et al. Towards wafer-size graphene layers by atmospheric pressure graphitization of silicon carbide. Nat. Mater. 8, 203-207 (2009).

17. Avouris, P. \& Dimitrakopoulos, C. Graphene: synthesis and applications. Mater. Today 15, 86-97 (2012).

18. Bedell, S. W. et al. Kerf-less removal of Si, Ge, and III-V layers by controlled spalling to enable low-cost PV technologies. IEEE J. Photovoltaics 2, 141-147 (2012)
19. Kim, J. et al. Layer-resolved graphene transfer via engineered strain layers. Science 342, 833-836 (2013).

20. Chierchia, R. et al. Microstructure of heteroepitaxial GaN revealed by X-ray diffraction. J. Appl. Phys. 93, 8918-8925 (2003).

21. Tasco, V. et al. Investigation of different mechanisms of $\mathrm{GaN}$ growth induced on AlN and GaN nucleation layers. J. Appl. Phys. 105, 063510 (2009).

22. Hong, W. et al. Persistent step-flow growth of strained films on vicinal substrates. Phys. Rev. Lett. 95, 095501 (2005).

23. Ratsch, C. \& Zangwill, A. Step-flow growth on strained surfaces. Appl. Phys. Lett. 63, 2348-2350 (1993).

24. Marchand, H. et al. Mechanisms of lateral epitaxial overgrowth of gallium nitride by metalorganic chemical vapor deposition. J. Cryst. Growth 195, 328-332 (1998).

25. Reitmeier, Z. J. et al. Surface and defect microstructure of GaN and AlN layers grown on hydrogen-etched $6 \mathrm{H}-\mathrm{SiC}(0001)$ substrates. Acta Mater. 58, 2165-2175 (2010).

26. Morkoc, Hadis. Handbook of nitride semiconductors and devices. Materials Properties, Physics, and Growth (Wiley-VCH, Verlag GmbH, 2008).

27. Bayram, C., Vashaei, Z. \& Razeghi, M. AlN/GaN double-barrier resonant tunneling diodes grown by metal-organic chemical vapor deposition. Appl. Phys. Lett. 96, 042103 (2010).

28. Niklaus, F., Stemme, G., Lu, J.-Q. \& Gutmann, R. J. Adhesive wafer bonding. J. Appl. Phys. 99, 031101 (2006).

29. Ueda, T., Ishida, M. \& Yuri, M. Laser lift-off of very thin AlGaN film from sapphire using selective decomposition of GaN interlayer. Appl. Surf. Sci. 216, 512-518 (2003).

30. Chu, C.-F. et al. Study of GaN light-emitting diodes fabricated by laser lift-off technique. J. Appl. Phys. 95, 3916-3922 (2004).

\section{Acknowledgements}

We thank E. Kim of Gearbox software for the assistance with figure illustration, $\mathrm{K}$. Fogel of IBM for the technical assistance with metal deposition, O. Gunawan of IBM for the support on setting up an electroluminescence system and M. Lu of IBM for EBSD measurement. J. Kim and H. Park thank J.B. Hannon of IBM for valuable technical discussion. C. Bayram thanks Prof. M. Razeghi, Director of Center for Quantum Devices of Northwestern University for her support.

\section{Author contributions}

J.K. conceived an idea. C.B. performed epitaxial growth. J.K. and C.B. designed the experiments. J.K., C.B. and H.P. performed fabrication/characterization of all samples and wrote the manuscript. H.P. performed LED measurements. C.-W.C. carried out XRD analysis and simulation. J.K. and C.D. performed graphitization of $\mathrm{SiC}$ substrates. J.A.O. and K.B.R. carried out TEM analysis. J.K., C.B., H.P., S.W.B. and D.K.S. participated in data analysis. All authors discussed the results and commented on the manuscript.

\section{Additional information}

Supplementary Information accompanies this paper at http://www.nature.com/ naturecommunications

Competing financial interests: The authors declare no competing financial interests

Reprints and permission information is available online at http://npg.nature.com/ reprintsandpermissions/

How to cite this article: Kim, J. et al. Principle of direct van der Waals epitaxy of single-crystalline films on epitaxial graphene. Nat. Commun. 5:4836 doi: 10.1038/ncomms5836 (2014). 